\title{
Abdominal symptoms as an initial manifestation of COVID-19 infection: Report of two cases
}

PATRÍCIA YOKOO ( $\square$ patyokoo@gmail.com )

Hospital Israelita Albert Einstein https://orcid.org/0000-0002-3493-8641

Eduardo Kaiser Ururahy Nunes Fonseca

Hospital Israelita Albert Einstein

Marcelo Oranges Filho

Hospital Israelita Albert Einstein

Rodrigo Caruso Chate

Hospital Israelita Albert Einstein

Gilberto Szarf

Hospital Israelita Albert Einstein

Ronaldo Hueb Baroni

Hospital Israelita Albert Einstein

\section{Case Report}

Keywords: COVID-19, coronavirus, abdominal pain, multidetector computed tomography

Posted Date: May 14th, 2020

DOl: https://doi.org/10.21203/rs.3.rs-28198/v1

License: (c) (i) This work is licensed under a Creative Commons Attribution 4.0 International License.

Read Full License 


\section{Abstract}

The novel coronavirus (COVID-19) pandemic started in December 2019 in Wuhan (Hubei, China) and spread rapidly; therefore, it is essential to detect the disease at an early stage and immediately isolate the infected patients [1]. The most common symptoms of COVID-19 infection include fever, asthenia, cough and dyspnea [2]. However, some patients are asymptomatic from the respiratory symptoms, and may only present abdominal manifestations as an initial finding, what creates a diagnostic challenge.

We describe two cases with diagnostic confirmations of COVID-19 who showed up at the Emergency Department with abdominal symptoms before presenting respiratory manifestations, and who had their initial suspicion based on the findings of the thoracoabdominal transition, demonstrating the importance of an adequate assessment of the lung base images.

\section{Case Presentation}

\section{Case 1}

A 55-year-old male patient was admitted to the emergency department with mild abdominal pain in the left iliac fossa 1 day ago. At that time he did not present any of the common symptoms: cough, fever, vomiting, diarrhea or shortness of breath. Upon physical examination, he reported abdominal discomfort in the left iliac fossa, with no changes in the respiratory system, 02 saturation of $97 \%$. A contrastenhanced abdominal computed tomography (CT) scan was performed in the portal venous phase, without significant abdominal findings (Fig 1). However, in the evaluation of the thoracoabdominal transition images, discrete ground-glass opacities were found on the periphery of the middle lobe and posterior basal segment of the right lobe. A complete chest CT was performed to better assess the extent and degree of pulmonary involvement, which corroborated the diagnosis of an infectious process of probable viral etiology, with findings compatible with those that have been described in pulmonary involvement by COVID-19 (Fig 2) [3]. This hypothesis was confirmed by a positive real time polymerase chain reaction for COVID-19, obtained by nasal secretion and oropharynx swab. The patient was discharged with prescription of symptomatic medications and home isolation for 14 days.

\section{Case 2}

Male patient, 84 years old, was admitted at the Emergency Department with abdominal discomfort, 6 days of fever and altered bowel habit with an episode of mild diarrhea, but without nausea, vomiting or respiratory symptoms. Upon physical examination, he had documented fever (38C), without other respiratory or abdominal complaints. A post-contrast CT scan of the abdomen in the portal venous phase was performed, without significant abdominal findings (Fig 3). However, evaluation of the pulmonary bases revealed ground-glass opacities some of them associated with smooth thickening of the 
interlobular septa (crazy-paving), scattered across the lung parenchyma. A chest CT was performed to better assess the findings, which showed multiple ground-glass and crazy-paving pulmonary opacities in multifocal, bilateral, predominantly peripheral and posterior distribution, especially in the lower lobes, which are strongly suggestive of COVID-19 infecction (Fig 4) [3]. Laboratory tests showed an increased inflammatory serum biomarker (C-reactive protein, $32 \mathrm{mg} / \mathrm{L}$ ), and all other blood counts were normal. PCR for COVID-19 was positive from an oropharynx swab. Due to the risk factors of advanced age and arterial hypertension, hospitalization was chosen.

\section{Discussion}

The novel coronavirus pandemic (COVID-19) started in December 2019 in Wuhan (Hubei, China) and spread rapidly, with early detection and isolation as essential measures against this agent [1].

Retrospective studies from Wuhan, China indicated that the most frequent clinical manifestations are fever, cough and dyspnea. Less common symptoms are headache, hemoptysis and gastrointestinal involvement. At the beginning of the pandemic, abdominal symptoms were not usually present, including diarrhea $(2 \%-10.1 \%)$, nausea and vomiting $(1-3.6 \%)[4]$.

However, we observed that, in some patients, respiratory symptoms may be absent and they may present only abdominal manifestations as an initial finding, creating a diagnostic challenge. In this context, imaging exams, especially computed tomography, are important auxiliary tools, especially in the context of emergency care to reach the diagnosis.

Abdominal complaints are frequently assessed by imaging studies and most of the protocols include images of the pulmonary bases, a frequent site of pulmonary involvement in COVID-19. The most frequent findings in the pulmonary parenchyma include ground-glass opacities, consolidation and crazypaving with multifocal, bilateral distribution, predominantly peripheral and posterior, especially in the lower lobes $[3,5]$

In conclusion, it is estimated that some patients with COVID-19 infection do not show respiratory symptoms, which makes a diagnostic suspicion more difficult. It is known that abdominal symptoms are not uncommon in patients with COVID-19 and can appear early $[4,6]$. Thus, in the current context of a pandemic, it is important that radiologists maintain a high degree of suspicion, even in studies not directed at the chest in order to allow an early diagnosis of COVID-19 infection. Such findings also have an impact on the isolation measures that need to be put in order to reduce the transmission of the disease.

\section{Declarations}

Competing interests: The authors declare no competing interests. 
Conflict of interest and sources of funding: The authors declared no potential conflicts of interest of received financial support from third parties with respect to the research, authorship, and/or publication of this article.

Ethical approval: Ethical approval for this study was obtained from Comissão Nacional de Ética em pesquisa- APPROVAL NUMBER/ID: 3.954.553 (Attachment).

Author Contributions: Study Concept and Design: Patrícia Yokoo and Eduardo Kaiser Ururahy Nunes Fonseca. Acquisition, analysis and interpretation of data: Marcelo Oranges Filho and Ronaldo Hueb Baroni. Drafting of the Manuscript: Patrícia Yokoo and Eduardo Kaiser Ururahy Nunes Fonseca. Critical revision of the manuscript for important intellectual content: Ronaldo Hueb Baroni, Rodrigo Caruso Chate and Gilberto Szarf.

Consent to participate: The patient has consented to the submission of the case report for submission to the journal.

Consent to publish: The participants have consented to the submission of the case report to the journal.

Authors affiliation:

All from Diagnostic Imaging Department, Hospital Israelita Albert Einstein, São Paulo - Brazil Av. Albert Einstein, 627 - Postal code: 05652-901 - Phone +55 (11) 2151-0195

Corresponding author:

Patrícia Yokoo, MD

Imaging Department, Hospital Israelita Albert Einstein, São Paulo - Brazil Av. Albert Einstein, 627 Postal code: 05652-901 - Phone/FAX +55 (11) 2151-0195

Email: patyokoo@gmail.com

Author identifying information:

Patrícia Yokoo ORCID number 0000-0002-3493-8641

\section{References}

1. Novel coronavirus-China. Jan 12, 2020[EB/OL]. http://www.who.int/csr/don/12-january-2020-novelcoronavirus-china/en/. Accessed Jan 19, 2020.

2. Chen N, Zhou M, Dong X, et al (2020) Epidemiological and clinical characteristics of 99 cases of 2019 novel coronavirus pneumonia in Wuhan, China: a descriptive study. Lancet 395(10223):507513.

3. Kanne, J. P. (2020). Chest CT Findings in 2019 Novel Coronavirus (2019-nCoV) Infections from Wuhan, China: Key Points for the Radiologist. Radiology 295:16-17

4. Huang C, Wang Y, Li X, et al (2020) Clinical features of patients infected with 2019 novel coronavirus in Wuhan, China. Lancet 395:497-506 
5. Chung M, Bernheim A, Mei X, et al (2020) CT imaging features of 2019 novel coronavirus (2019nCoV). Radiology 295:202-207

6. Gao QY, Chen YX, Fang JY (2020) 2019 novel coronavirus infection and gastrointestinal tract. J Dig Dis. http:// doi:10.1111/1751-2980.12851

\section{Figures}

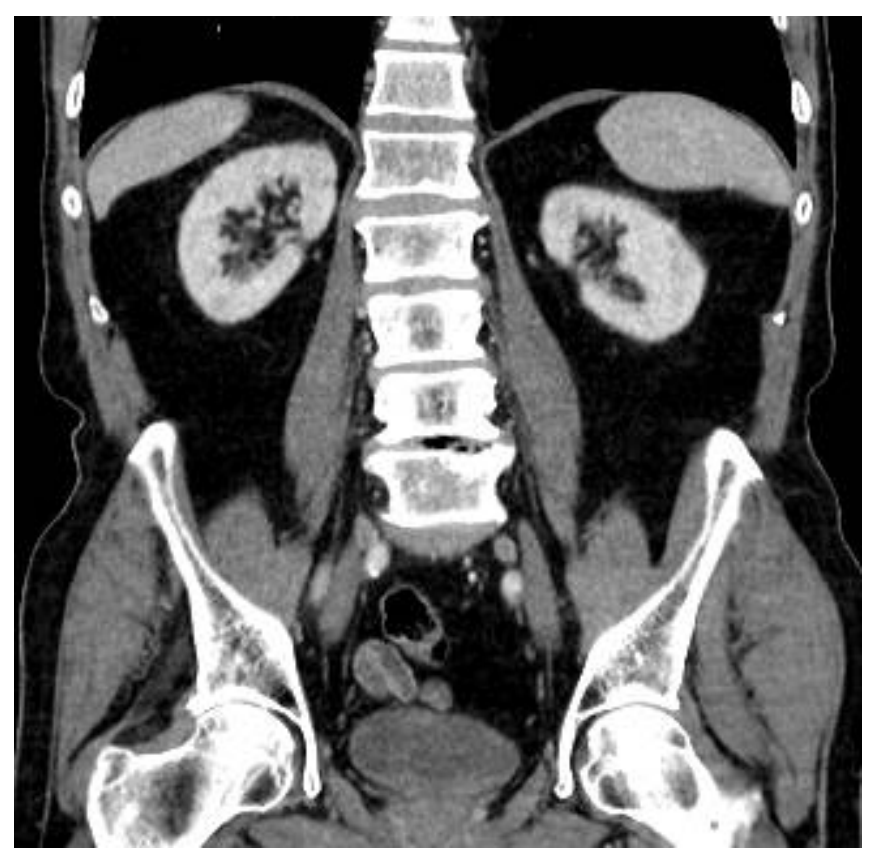

Figure 1

Contrast-enhanced abdominal CT in the portal venous phase, without significant abdominal findings
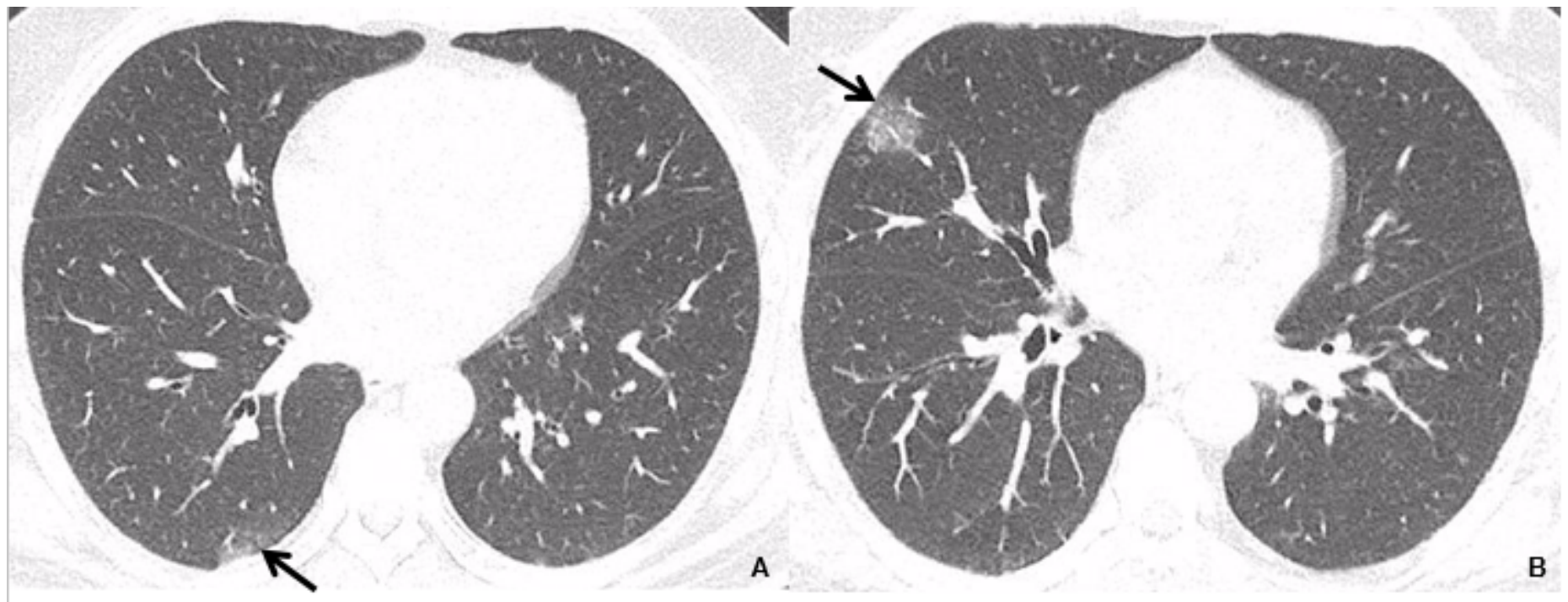

Figure 2 
Unenhanced CT images shows ground-glass opacities in bilateral lungs (arrows). Ground-glass opacities are seen in the posterior segment of right lower lobe $(A)$ and in the periphery of the middle lobe (B)

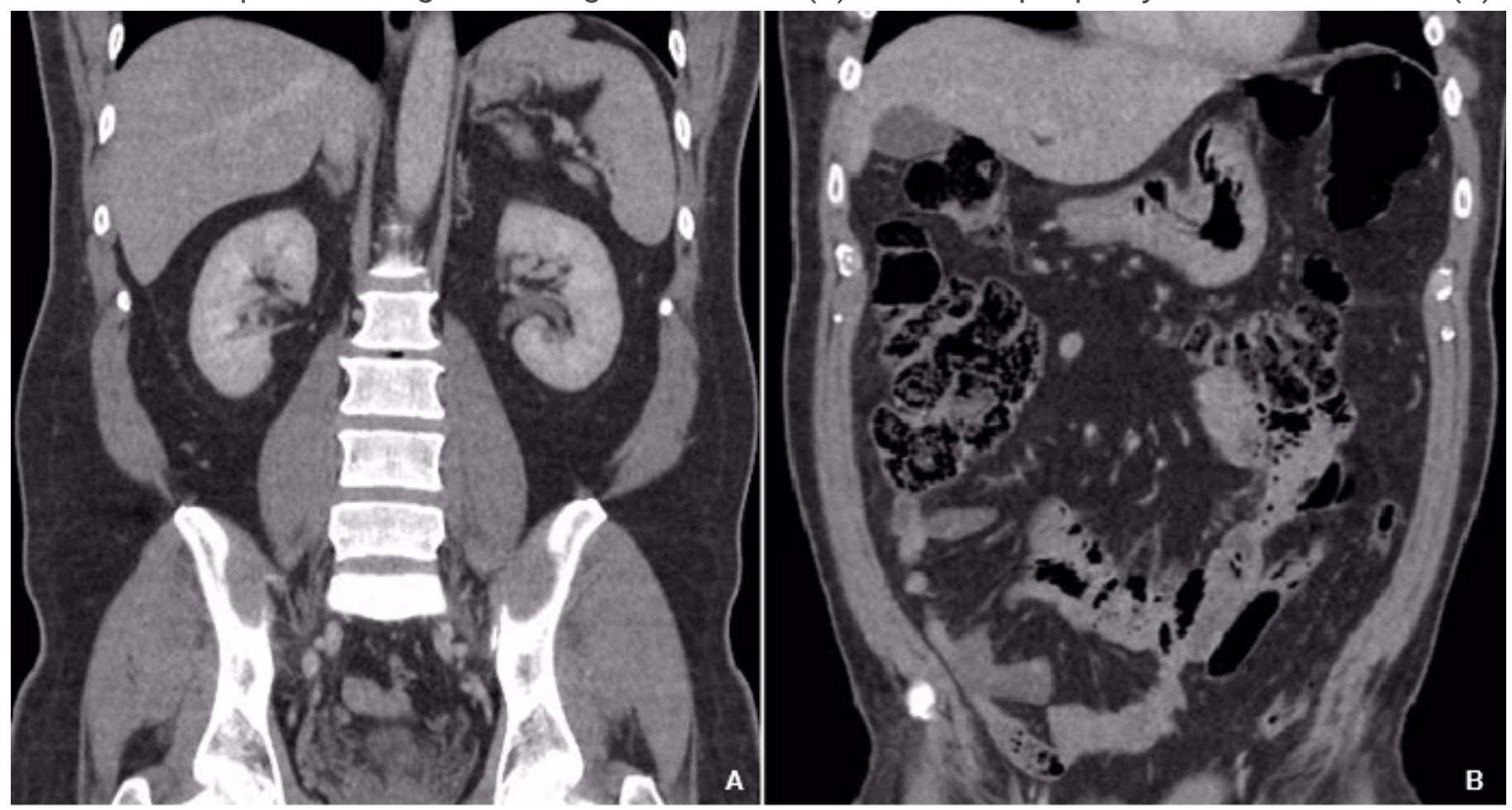

Figure 3

Contrast-enhanced abdominal CT in the portal venous phase, without significant abdominal findings 


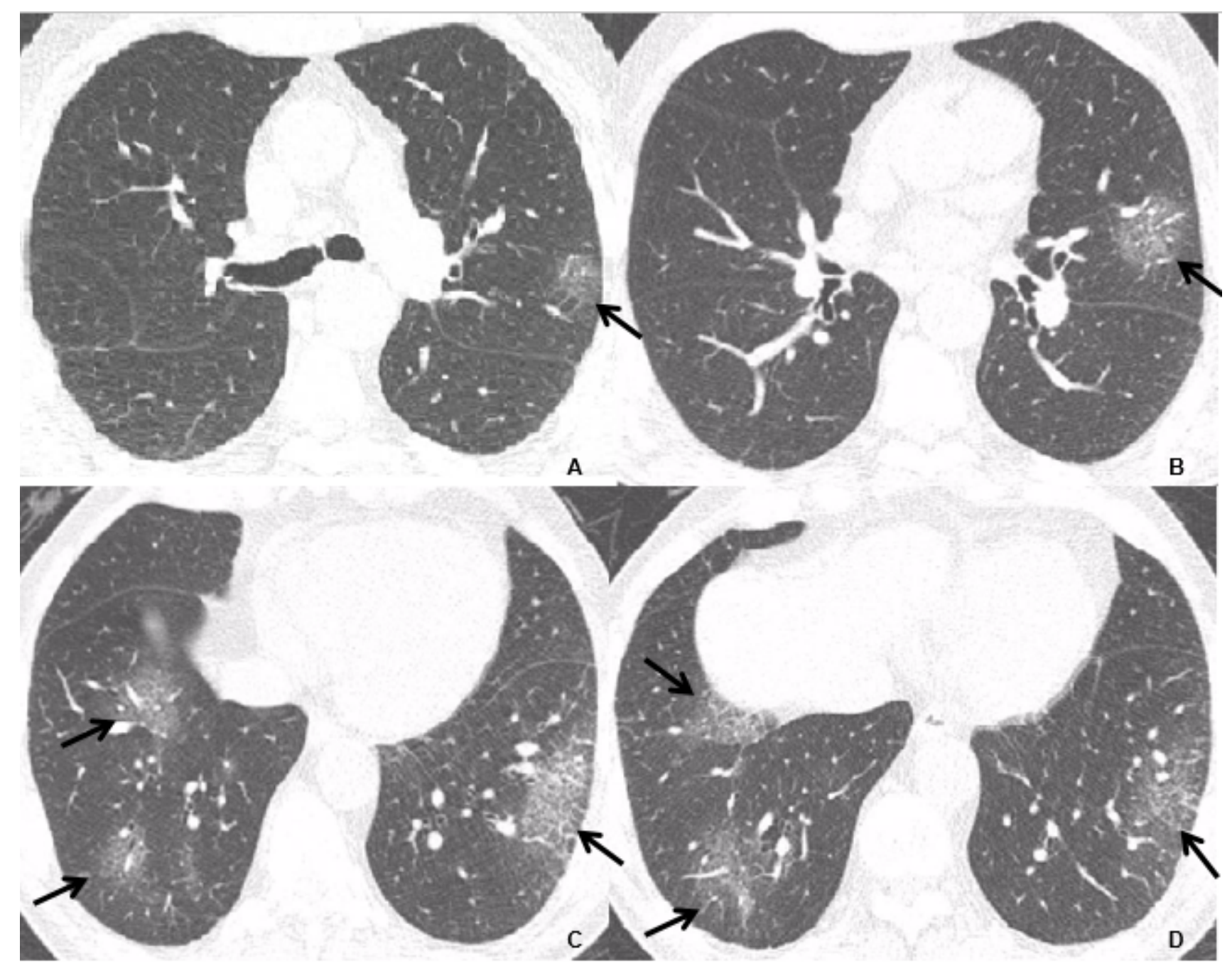

Figure 4

Unenhanced CT images shows multiple ground-glass and crazy-paving pulmonary opacities (arrows) in multifocal, bilateral, predominantly peripheral and posterior distribution (A-D), especially in the lower lobes $(C, D)$ 